\title{
O impacto de medidas restritivas devido à pandemia por COVID-19 nas concentrações de poluentes atmosféricos em cidade de médio porte
}

\section{The impact of restrictive measures due to the COVID-19 pandemic on air pollutant concentrations in a medium-sized city}

\author{
Igor Soares Mantovani ${ }^{1}$, Andressa Ferreira Pimenta ${ }^{1}$, Isadora Caroline Bieleski ${ }^{1}$, Alexandra \\ Beal $^{2}$, Leila D. Martins ${ }^{2}$, Maria Cristina Solci ${ }^{*}$
}

\begin{abstract}
RESUMO
O avanço da pandemia provocada pela COVID-19, além de causar danos à saúde humana, também apresentou efeitos indiretos na qualidade do ar, apresentando melhorias significativas. A relação entre a transmissibilidade do vírus SARS-Cov-2 e a poluição do ar suscita a preocupação em estudar o impacto das medidas de proteção contra o avanço da doença na qualidade do ar. As coletas de material particulado (MP) foram realizadas em abril de 2020, período em que foram observadas medidas de restrição à circulação de veículos e sua relaxação, utilizando amostradores tipo ciclone de baixo volume para as frações $\mathrm{MP}_{1,0}$ e $\mathrm{MP}_{2,5}$. A quantificação do Black Carbon foi realizada por meio do reflectômetro (EEL 43M). As medidas adotadas para combater a COVID-19, por reduzirem fontes de emissão, contribuíram diretamente para a melhoria da qualidade do ar, resultando em uma diminuição na concentração de todos os analitos estudados neste trabalho. Houve uma variação na concentração de $\mathrm{MP}_{1,0}$ e $\mathrm{MP}_{2,5}$ de $31,5 \%$ e $27,4 \%$ respectivamente em MHL e 40,1\% e 34,2\% em TOU. As medidas adotadas para conter o avanço da COVID-19 contribuíram diretamente para a melhora na qualidade do ar.
\end{abstract}

Palavras-chave: Material particulado fino; Black Carbon; Pandemia; COVID-19

\section{ABSTRACT}

The advance of the pandemic caused by COVID-19, in addition to causing damage to human health, also had indirect effects, such as air quality, which in major cities showed significant improvements. The relationship between the transmissibility of the SARS-Cov-2 virus and air pollution raises concerns about studying the impact of protective measures against the spread of the disease on air quality. The collections of particulate matter (PM) were carried out in April 2020, a period in which measures were observed to restrict the circulation of vehicles and their relaxation, using low volume cyclone samplers for the $\mathrm{MP}_{1.0}$ and $\mathrm{MP}_{2.5}$ fractions. The quantification of Black Carbon was performed by means of a reflectometer (EEL $43 \mathrm{M})$. The measures adopted to combat COVID-19, by reducing emission sources, contributed directly to the improvement of air quality, resulting in a decrease in the concentration of all analytes studied in this work. There was a variation in the concentration of $\mathrm{PM}_{1.0}$ and $\mathrm{PM}_{2.5}$ of $31.5 \%$ and $27.4 \%$ respectively, in MHL and $40.1 \%$ and $34.2 \%$ in TOU. The measures adopted to curb the advancement of COVID-19 contributed directly to the improvement in air quality.

Keywords: Fine Particulate Material; Black Carbon; Pandemic; COVID-19

\footnotetext{
${ }^{1}$ Departamento de Química, Universidade Estadual de Londrina, Londrina/PR, Brasil

${ }^{2}$ Universidade Tecnológica Federal do Paraná, Londrina/PR, Brasil

*E-mail: solci@uel.br
} 


\section{INTRODUÇÃO}

Desde que o surto de COVID-19 foi decretado pela Organização Mundial da Saúde (OMS) em 11 de março de 2020 como pandemia (BARRETO, 2020), medidas sanitárias e de restrição à mobilidade foram adotadas por autoridades governamentais a fim de minimizar a propagação da doença. Os efeitos causados pela pandemia de COVID19 no mundo, que além de impactar diretamente na saúde das pessoas, causaram efeitos indiretos, como na qualidade do ar que apresentou uma melhora significativa logo após a propagação da doença, em grandes centros urbanos. As emissões diárias globais de dióxido de carbono $\left(\mathrm{CO}_{2}\right)$ diminuíram 17\% no início de abril de 2020 em comparação com os valores médios de 2019 (LE QUÉRÉ et al., 2020). No Brasil, de acordo com dados oficiais, o primeiro caso de COVID-19 foi reportado em 26 de fevereiro de 2020 e o estado de São Paulo foi o primeiro a adotar medidas restritivas levando consequentemente à queda expressiva da mobilidade (caminhada e direção) no período de 16 a 23 de março. A redução da mobilidade e aumento da taxa de isolamento influenciaram nas concentrações de poluentes atmosféricos na cidade de São Paulo (NAKADA e URBAN, 2020). Houve redução de 30\% nas concentrações de monóxido de carbono (CO), $40 \%$ de monóxido de nitrogênio (NO), $22 \%$ em dióxido de nitrogênio $\left(\mathrm{NO}_{2}\right), 26 \%$ em óxidos de nitrogênio $\left(\mathrm{NO}_{\mathrm{x}}\right)$ e somente $0,3 \%$ de material particulado fino $\left(\leq \mathrm{MP}_{2,5}\right)$. No entanto, as concentrações de material particulado grosso $\left(\mathrm{MP}_{10}\right)$ e ozônio $\left(\mathrm{O}_{3}\right)$ cresceram 7,7 e $11 \%$, respectivamente. Na cidade do Rio de Janeiro houve diminuição nas concentrações dos poluentes, entretanto esta variação não foi proporcional à redução do fluxo de veículos (DANTAS et al., 2020).

É importante ressaltar que diversos estudos têm associado a poluição atmosférica a danos à saúde humana, como doenças respiratórias, cardiovasculares (NARDOCCI et al., 2020), doenças mutagênicas e diabetes tipo-2 (RAJAGOPALAN e BROOK, 2012) sendo ainda a poluição atmosférica apontada como principal fator de risco na incidência de doenças respiratórias em crianças (BEBER et al., 2020). A poluição atmosférica foi associada à transmissão e gravidade da COVID-19, observando que regiões com maiores concentrações de $\mathrm{MP}_{10}$ e $\mathrm{MP}_{2,5}$ foram mais afetadas pela doença viral que causa síndrome respiratória aguda (DOMINGO e ROVIRA, 2020). O MP pode operar como transportador de vírus, o que permite maior difusão e permanência no ar, além de alterar a imunidade do hospedeiro para infecções virais respiratórias (MARTELLETTI e MARTELLETTI, 2020). O estudo combinado de dados de satélite da exposição a 
partículas finas contaminantes $\left(\mathrm{MP}_{2,5}\right)$ e dados de redes de vigilância e contaminação dos solos mostrou que a exposição à longo prazo à poluição atmosférica pode aumentar o risco por morte por COVID-19 em média de 15\% no mundo. A exposição à poluição do ar teria contribuído com 12\% das mortes por COVID-19 no Brasil, 29\% na República Tcheca, 27\% na China, 26\% na Alemanha, 22\% na Suíça, 21\% na Bélgica, 19\% na Holanda, 18\% na França, 15\% na Itália, 14\% no Reino Unido, 11\% em Portugal, 9\% na Espanha, 6\% em Israel, 3\% na Austrália e 1\% na Nova Zelândia (LELIEVELD et al., 2020).

No Paraná, o primeiro caso de COVID-19 foi reportado em Curitiba em 11 de março de 2020 e em Londrina, em 17 de março (SINIMBÚ, 2020). Na mesma data, medidas de contenção foram implementadas, com a suspensão das aulas da UEL (UNIVERSIDADE ESTADUAL DE LONDRINA, 2020) e em 20 e 23 de março houve a suspenção das aulas presenciais na rede estadual e municipal de ensino, respectivamente. Em 23 de março teve início a restrição aos estabelecimentos comerciais, obrigando-os a permanecerem fechados e as indústrias permaneceram em funcionamento até o dia 27 de março, sendo por decreto municipal fechadas a partir desta data. Estas ações impactaram diretamente no índice de mobilidade urbana, durante o período de quarentena, que no estado do Paraná diminuiu em média de $45 \%$ (APPLE MOBILITY TRENDS, 2020).

Londrina é caracterizada como cidade de médio porte com população estimada em 569.733 habitantes (IBGE, 2019). São escassos os dados oficiais que apresentem a variação temporal e espacial de poluentes atmosféricos legislados. Alguns trabalhos foram desenvolvidos na cidade mostrando que os principais poluentes atmosféricos foram $\mathrm{MP}$ e óxidos de enxofre $\left(\mathrm{SO}_{\mathrm{x}}\right)$, provenientes predominantemente de fontes industriais e $\mathrm{CO}$ e NOx provenientes de fontes veiculares (IAP, 2013). Black Carbon (BC), $\mathrm{NO}_{\mathrm{x}} \mathrm{e}$ $\mathrm{MP}_{2,5}$ foram medidos em Londrina apresentando resultados de maiores concentrações em área central da cidade quando comparado com a periferia (KRECL et al., 2019). Estudo realizado visando a distribuição sazonal de $\mathrm{MP}_{2,5}$ e $\mathrm{BC}$ em área urbana de Londrina no período de maio 2017 a julho de 2018 mostrou concentrações elevadas de partículas finas com média de 35,4 $\mu \mathrm{g} \mathrm{m}^{-3}$ e BC de 1,7 $\mu \mathrm{g} \mathrm{m}^{-3}$ (SANTOS et al., 2020).

Uma peculiaridade da pandemia é marcada pela variabilidade regional na difusão do vírus e da gravidade das manifestações clínicas da doença (MANIGRASSO et al., 
2020). Diante da urgência em compreender a transmissibilidade do vírus SARS-Cov-2 e a relação com emissões poluentes, este trabalho teve como objetivo avaliar as mudanças na qualidade do ar causadas pelas medidas de contenção da COVID-19.

\section{METODOLOGIA}

\section{Área de estudo e os locais de amostragem}

O estudo foi realizado em sítios localizados na cidade de Londrina, que compõe a mesorregião norte central paranaense, localizada no norte do Paraná, sul do Brasil. Foram realizadas amostragens no pátio externo do Museu Histórico de Londrina (MHL), localizado no centro da cidade $\left(23^{\circ} 18 ' 29.30^{\prime \prime} \mathrm{S}, 51^{\circ} 9^{\prime} 34.39^{\prime \prime O}\right)$. O Museu localiza-se em uma avenida com alto tráfego veicular, ao lado do terminal de ônibus urbano (TOU). Paralelamente, foram realizadas amostragens no interior do terminal, onde há somente a circulação de ônibus. É um local semifechado, com baixa circulação de ar cuja velocidade média dos ônibus é de $20 \mathrm{~km} \mathrm{~h}^{-1}$. As amostragens foram realizadas no piso térreo onde os ônibus transitam por curto espaço de tempo. O terceiro ponto de amostragem foi na Universidade Tecnológica Federal do Paraná (UTFPR) localizada na região leste da cidade (2318”26.49”S, 5106'53.48”O). Trata-se de um lugar mais afastado da área central, com pouca circulação de veículos.

\section{Metodologia de amostragem}

O período de amostragem ocorreu de 2 a 25 de abril de 2020. Neste período foram observadas medidas de restrição de circulação veicular assim como o seu relaxamento. Para as coletas foram utilizados em paralelo, dois amostradores ciclones de baixo volume para MP2,5 (URG-2000-30EH, URG® Corporation, USA) e MP1,0 (URG-200030EHB, URG® Corporation, USA), que foram conectados a bomba de vácuo por $24 \mathrm{~h}$. Válvulas de agulha foram utilizadas para o controle da vazão mantida em $16,7 \mathrm{~L} \mathrm{~min}^{-1}$. Foram utilizados filtros de fibra de quartzo com $47 \mathrm{~mm}$ de diâmetro. Antes das coletas, os filtros foram submetidos à tratamento térmico por $3 \mathrm{~h}$ em mufla a $400{ }^{\circ} \mathrm{C}$ para retirada de possíveis interferentes. Os filtros foram mantidos em sala condicionada com temperatura e umidade relativa controladas em $24 \pm 3^{\circ} \mathrm{C}$ e $30 \pm 2 \%$, respectivamente, por $24 \mathrm{~h}$ antes e após a coleta (DA SILVA et al., 2018).

\section{Análise gravimétrica}


Para a análise gravimétrica, os filtros foram pesados antes e após a coleta em balança ultra-analítica (Mettler Toledo, Modelo AX26) com precisão de 1,0 $\mu \mathrm{g}$. A massa do MP foi calculada com base na diferença entre as massas iniciais e finais dos filtros. Após a análise gravimetria, os filtros foram embalados em papel alumínio previamente tratado termicamente e armazenados em embalagem plástica sob refrigeração.

\section{Black carbon (BC)}

Na UTFPR as concentrações de BC foram obtidas a partir de aetalômetro, modelo AE42 (Magee Scientific, Berkeley, CA, EUA) na vazão de $5 \mathrm{~L} \mathrm{~min}^{-1}$ e com entrada selecionada de 2,5 $\mu \mathrm{m}$. Nas amostras em MHL e TOU, a concentração de BC foi determinada por meio do método de refletância da luz, com o auxílio de reflectómetro EEL 43D (DiffusionSystems Ltd., London, UK). A concentração de BC foi obtida a partir do valor de refletância, área do filtro e volume da amostra. A luz refletida é inversamente proporcional à luz absorvida, que é função da quantidade de material absorvente presente na amostra (DA SILVA et al., 2018).

\section{Mobilidade urbana, dados meteorológicos e casos acumulados de COVID-19}

Os dados de mobilidade urbana foram obtidos a partir dos relatórios de tendências de movimentação fornecidos pela Apple e pelo Google. Os dados são gerados a partir de solicitações feitas pelos usuários ao sistema de mapas da fabricante para obter rotas e pela variação no tempo de permanência em locais diferentes mudam em comparação com um valor base (APPLE, 2020; GOOGLE, 2021). Os dados meteorológicos foram disponibilizados pelo Instituto Nacional de Meteorologia (INMET) e pelo Instituto de Desenvolvimento Rural do Paraná, por meio do aplicativo IAPAR Clima que fornece os dados ao público geral (IAPAR, 2020; INMET, 2020). Dados de casos acumulados de COVID-19, assim como o número de óbitos causados pela doença, foram obtidos pelo portal “Corona vírus Brasil” (MINISTÉRIO DA SAÚDE, 2021).

\section{RESULTADOS E DISCOSSÃO}

\section{A evolução no número de casos e óbitos por COVID-19 em Londrina}

As Figuras 1 e 2 apresentam os números diários de casos de COVID-19 e o de mortes pela doença, respectivamente, assim como a média móvel para um período de 15 
dias. Os dados foram analisados no período de 27 de março de 2020 a 27 de março de 2020, na cidade de Londrina-PR, destacando no gráfico o período de 27 de março a 27 de maio de 2020. Os casos de COVID-19 começaram a aumentar em maio quando houve um relaxamento nas medidas de segurança com a abertura das indústrias e posteriormente do comércio.

Figura 1: Casos diários de COVID-19 e média móvel de casos no período de 27 de março de 2020 a 27 de março de 2021 em Londrina-PR

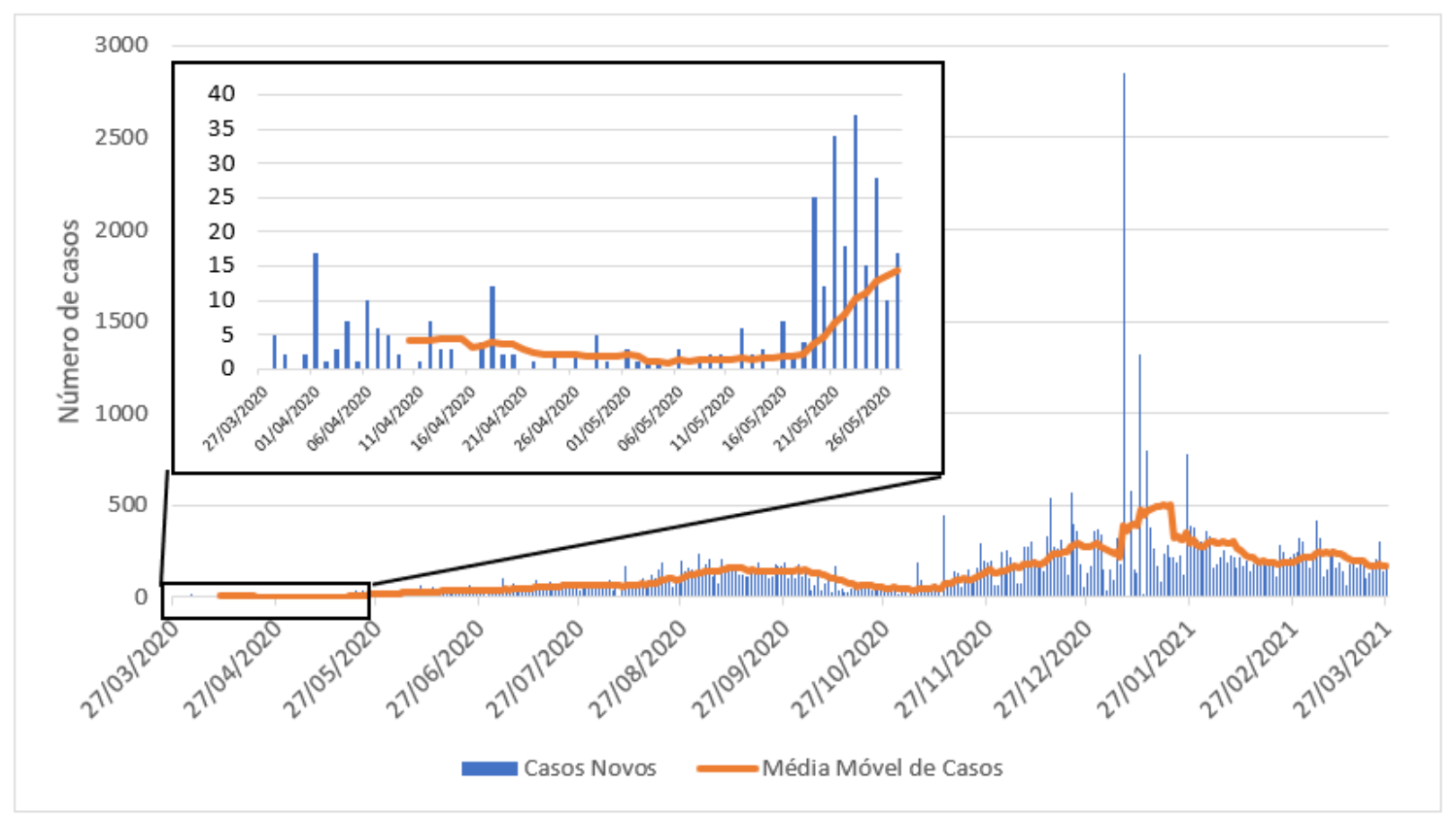

Fonte: Ministério da Saúde (2021) 
Figura 2: Número diário de óbitos e média móvel causadas pela COVID-19 no período de 27 de março de 2020 a 27 de março de 2021 em Londrina-PR

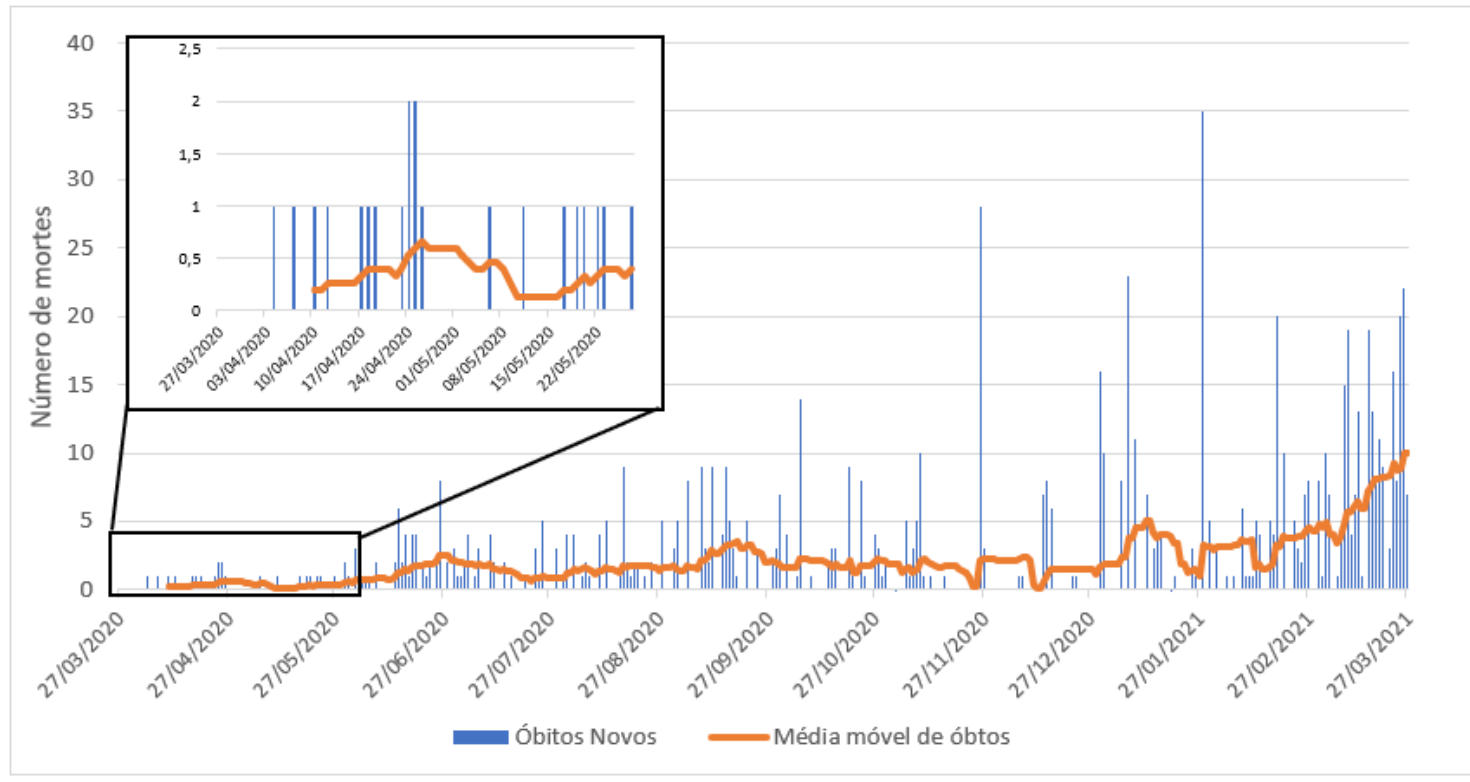

Fonte: Ministério da Saúde (2021)

\section{Mobilidade urbana}

Os dados de mobilidade urbana para o Estado do Paraná foram obtidos para o período de janeiro a outubro de 2020 e podem ser verificados na Figura 3. São apresentados os dados referentes à mobilidade com uso de automóveis, para pessoas em caminhada e a taxa de referência. Observa-se a diminuição da mobilidade urbana em torno de $41 \%$ em 15 de março coincidente com a data de restrição implementada no Estado alcançando 70 e $80 \%$ em 22 de março com o uso de veículos e pedestres, respectivamente. A taxa de mobilidade foi aumentando ao longo dos meses mostrando o relaxamento das medidas de restrição. Para pedestres, a taxa de mobilidade teve um aumento menos acentuado comparado com pessoas motorizadas.

Observa-se na Figura 4, que houve um aumento de $25 \%$ na permanência em locais residenciais, mantendo-se com aumento médio de $10 \%$ até dezembro, significando que as pessoas permaneciam em suas residências. Os locais de trabalho tiveram a redução de $45 \%$ de permanência no período que coincide com as restrições impostas pela administração municipal. Entretanto, com o retorno das atividades comerciais e industriais, a taxa de permanência voltou a apresentar valores próximos à média de $0 \%$. Estações de transporte público e locais de varejo e lazer tiveram redução de permanência das pessoas de $80 \%$ e $60 \%$, respectivamente, mantendo a média de $20 \%$ até o final do ano. Em parques, este valor foi mantido como média para todo o período de 2020, mesmo 
com o fim das ações restritivas, mostrando que parte da população manteve as ações de prevenção mesmo depois do fim das limitações. Os mercados e farmácias tiveram leve diminuição no período em que as restrições estavam vigentes, entretanto, com o fim das mesmas começaram a apresentar um aumento constante, obtendo aproximadamente $50 \%$ em dezembro.

Figura 1: Taxa de mobilidade urbana para o Estado do Paraná

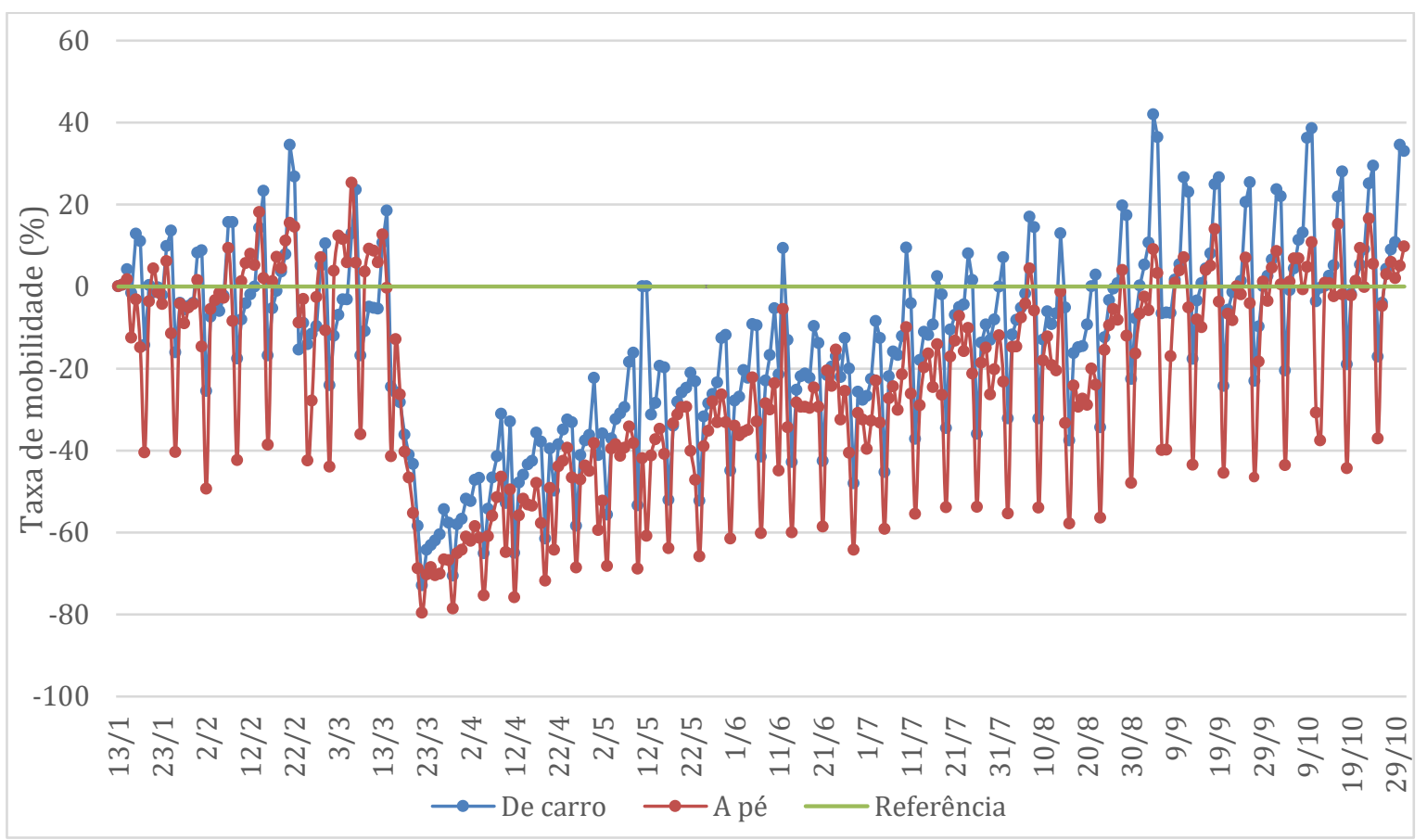

Fonte: Apple Mobility Trends (2020) 
Figura 4: Variação da mobilidade urbana em diferentes tipos de lugar, para a cidade de Londrina-PR

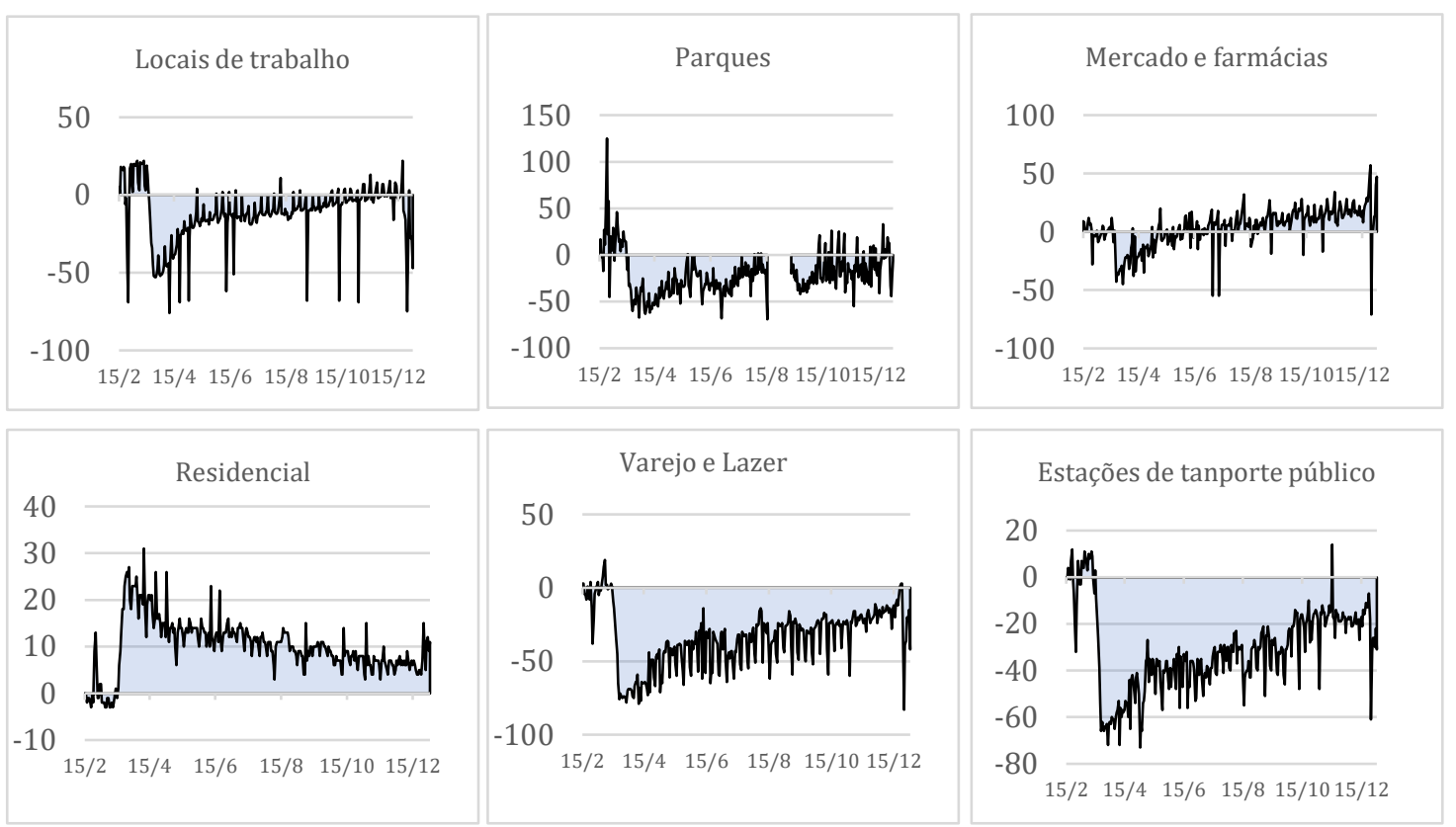

Fonte: GOOGLE (2020).

\section{Dados meteorológicos}

No período de amostragem as condições meteorológicas se mantiveram estáveis com temperaturas variando de $9,2{ }^{\circ} \mathrm{C}$ a $33,3{ }^{\circ} \mathrm{C}$. Foram observadas precipitações apenas nos dias 06 e 14 de abril, apesar da pouca chuva, a umidade relativa do ar se manteve elevada, variando de $56 \%$ a $85 \%$. Pode-se observar que o fator climático não foi decisivo para o aumento na concentração do MP.

\section{Concentração de MP fino $\left(\mathrm{MP}_{2,5}\right.$ e $\left.M P_{1,0}\right)$ e $\mathrm{BC}\left(\mathrm{BC}_{2,5}\right.$ e $\left.\mathrm{BC}_{1,0}\right)$}

No perído de amostragem compreendido entre os dias 02 e 24 de abril de 2020 foram obtidas 42 amostras no MHL e 39 amostas no TOU. Foram determinadas as concentrações de $\mathrm{MP}_{2,5}, \mathrm{MP}_{1,0}$ e $\mathrm{BC}$ para cada fração de tamanho $\left(\mathrm{BC}_{2,5}\right.$ e $\left.\mathrm{BC}_{1,0}\right)$. Os valores de $\mathrm{MP}_{2,5}$ e $\mathrm{MP}_{1,0}$ foram organizados nas Figuras 5 e 6. 
Figura 5: Concentração diária de $\mathrm{MP}_{2,5} \mathrm{e} \mathrm{MP}_{1,0} \mathrm{em} \mu \mathrm{g} \mathrm{m}{ }^{-3}$, no pátio do MHL no período de 02 a 25 de abril de 2020 em Londrina-PR

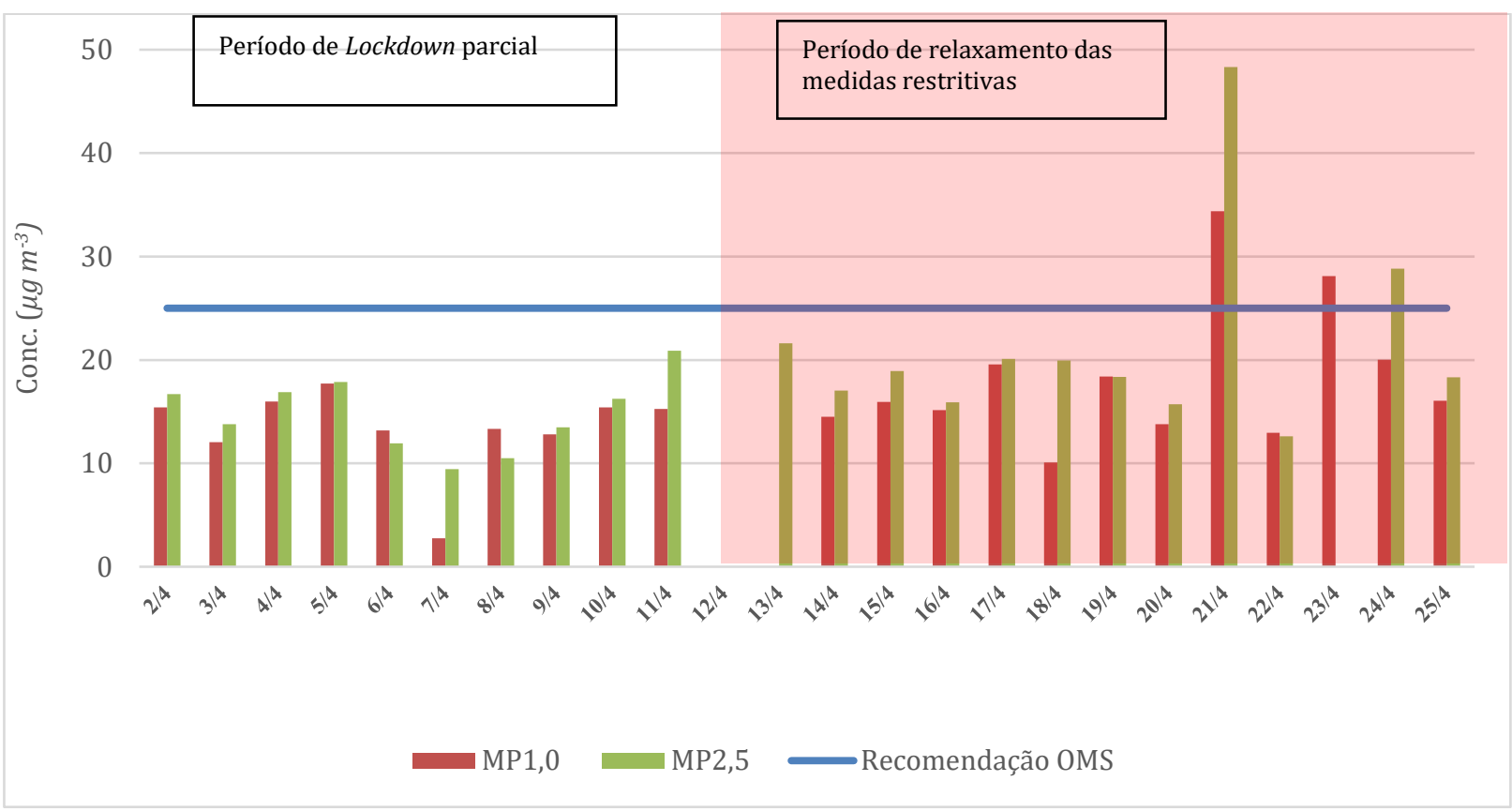

Fonte: Dos autores.

Figura 6: Concentração diária de $\mathrm{MP}_{2,5}$ e $\mathrm{MP}_{1,0} \mathrm{em} \mu \mathrm{g} \mathrm{m}{ }^{-3}$, no interior do TOU de Londrina

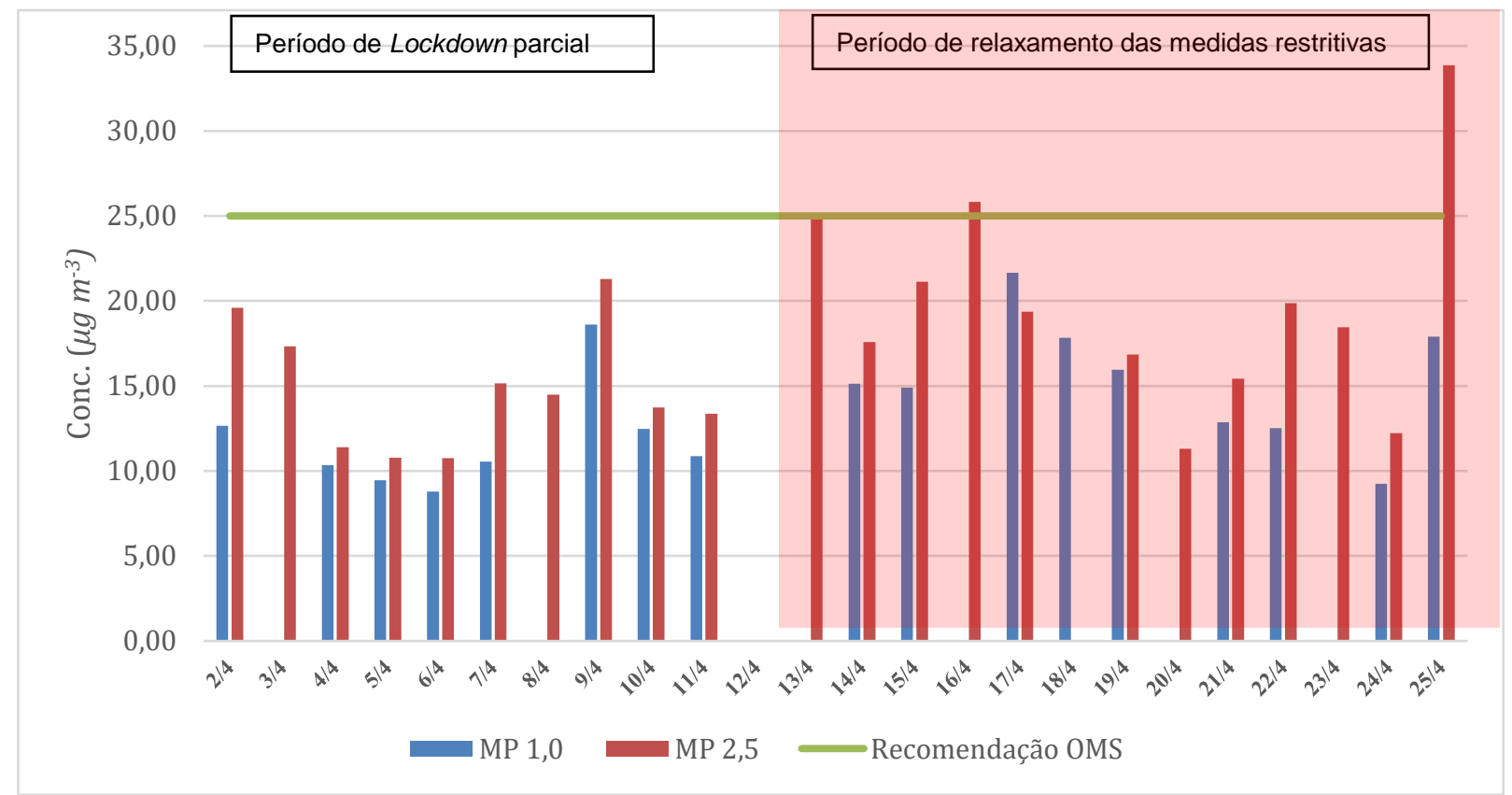

Fonte: Dos autores.

No MHL, as concentrações de $\mathrm{MP}_{2,5}$ variaram de 9,4 a 48,3 $\mu \mathrm{g} \mathrm{m}^{-3}$, com média no período de $18,3 \mu \mathrm{g} \mathrm{m}^{-3}$, enquanto o $\mathrm{MP}_{1,0}$ variou de 2,8 a $34,4 \mu \mathrm{g} \mathrm{m}^{-3}$, com média de 16,0 
$\mu \mathrm{g} \mathrm{m}^{-3}$ correspondendo a $87 \%$ do $\mathrm{MP}_{1,0}$ na fração fina. Resultados obtidos em amostras coletadas no mesmo local nos meses de março e abril de 2007 (FREITAS e SOLCI, 2009) mostraram concentração média de $17,5 \mu \mathrm{g} \mathrm{m}^{-3}$ de $\mathrm{MP}_{2,5}$.

As amostras do terminal urbano apresentaram concentrações variando de 10,8 a $33,9 \mu \mathrm{g} \mathrm{m}^{-3}$ para o $\mathrm{MP}_{2,5}$ e de 8,8 a $21,7 \mu \mathrm{g} \mathrm{m}^{-3}$ para o $\mathrm{MP}_{1,0}$, sendo 14,3 e $17,4 \mu \mathrm{g} \mathrm{m}^{-3}$ as concentrações médias de $\mathrm{MP}_{1,0}$ e $\mathrm{MP}_{2,5}$, respectivamente, e $82 \%$ de $\mathrm{MP}_{1,0}$ correspondente ao MP fino. Resultados obtidos em amostras coletadas no mesmo local ao longo de cinco campanhas de amostragem (2008 a 2017) acompanhando o acréscimo do biodiesel ao diesel de B3 a B8 (DA SILVA et al., 2018) mostraram que em média 83\% da fração fina correspondeu ao $\mathrm{MP}_{1,0}$. Em 2017 (B8) a concentração média de $\mathrm{MP}_{2,5}$ foi de 25,3 $\mu \mathrm{g} \mathrm{m}^{-3}$.

Ao considerar os períodos de restrição (02/04 a 11/04) e a subsequente reabertura das atividades comerciais e industriais (12/04 a 24/04), houve uma variação média da concentração do $\mathrm{MP}_{1,0}$ e $\mathrm{MP}_{2,5}$ de 31,5 e 27,4\%, respectivamente, no ambiente externo do MHL. No TOU a variação foi de 40,1\% para o $\mathrm{MP}_{1,0}$ e $34,2 \%$ para o $\mathrm{MP}_{2,5}$.

Considerando as médias das concentrações do MP fino, observa-se, em sua maioria, que os níveis não excedem os valores recomendados pela OMS no período de 24 horas $\left(25 \mu \mathrm{g} \mathrm{m}^{-3}\right)$. Valores superiores à recomendação da OMS aparecem no período após 12/04 com a reabertura do setor industrial da cidade. Esta intervenção pode ser atribuída à direção dos ventos nos dias de amostragem, que sopravam da região industrial para a região central da cidade.

$\mathrm{Na}$ UTFPR, foram realizadas somente medidas de $\mathrm{BC}$ na fração $\mathrm{MP}_{2,5}$. A Figura 7 mostra a variabilidade do $\mathrm{BC}$ relativo às frações $\mathrm{MP}_{1,0}$ e $\mathrm{MP}_{2,5}$ comparadas entre os dois locais de amostragem no período estudado. 
Figura 7: Concentração diária de BC no MHL e no campus da UTFPR, Londrina-PR no período de 02 a 24 de abril de 2020

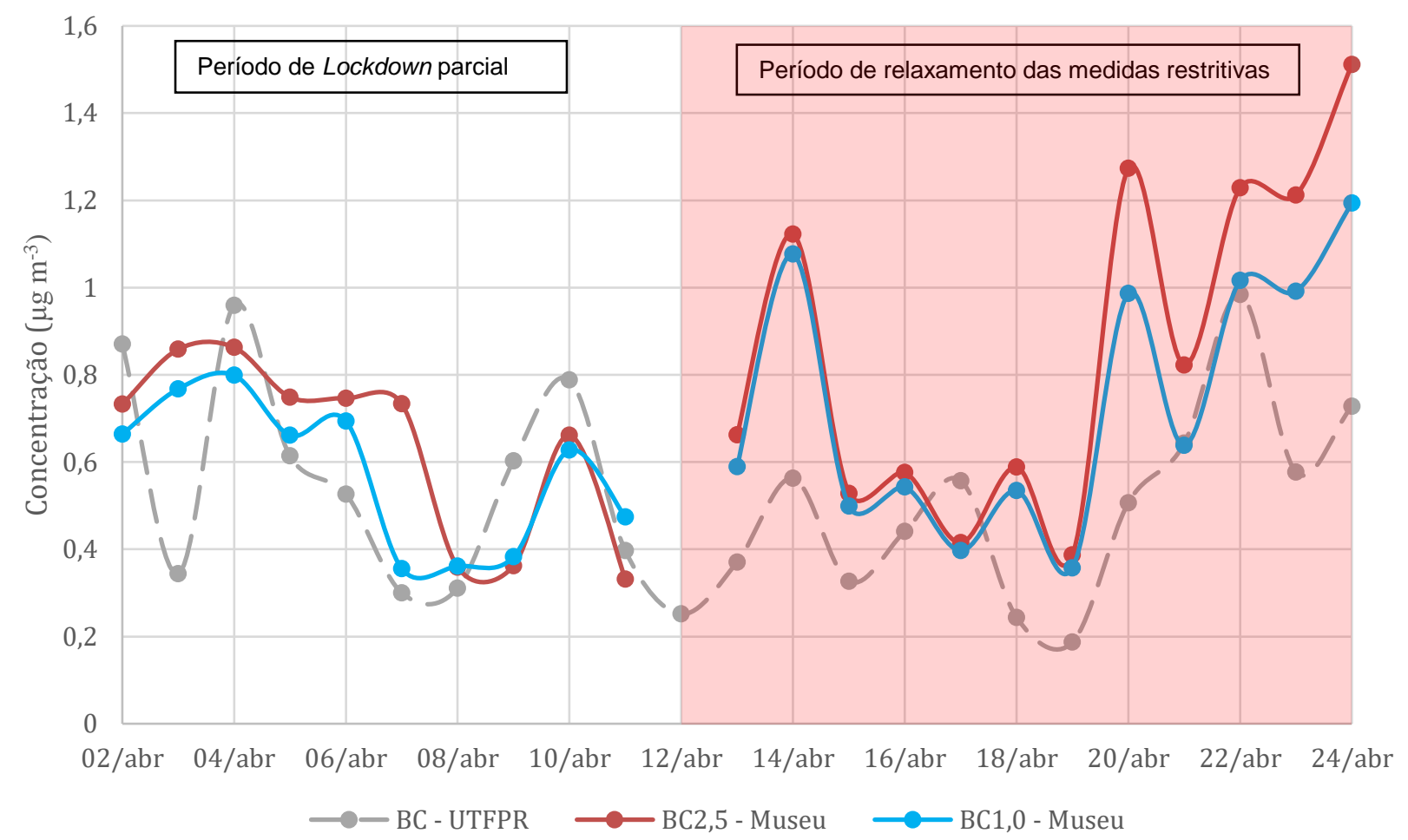

Fonte: Dos autores.

Por se tratar de um local mais afastado do centro da cidade, as concentrações de BC observadas no campus da UTFPR mostraram-se em média, menores que as observadas no MHL. Observa-se o aumento em BC quando do relaxamento da restrição. Da mesma forma, as concentrações de MP fino aumentaram mostrando a influência provável de fontes de combustão como emissoras de poluentes.

A Tabela 1 apresenta as faixas de concentração de BC no MP fino nos três pontos de amostragem no período. A fração $\mathrm{BC}_{1,0}$ correspondeu a $86 \%$ da fração fina do $\mathrm{MP}$ coletado no pátio do MHL. Em estudo prévio realizado em Londrina nos anos de 2013 e 2014 no mesmo local de amostragem (UTFPR) (BEAL et al., 2017) foram relatadas concentrações médias de BC com intervalo de concentração de 0,9 a 1,9 $\mu \mathrm{g} \mathrm{m}^{-3}$. Em 2020, no mesmo local foi obtido o intervalo de concentração de 0,19 a $0,98 \mu \mathrm{g} \mathrm{m}^{-3}$. A concentração de BC depende da intensidade das fontes de emissão, da dispersão e do transporte a longas-distâncias. Contribuição de fontes veiculares e queima de biomassa são fatores a serem considerados. 
Tabela 1: Concentrações de $\mathrm{BC}\left(\mu \mathrm{g} \mathrm{m}^{-3}\right)$ observadas nos três locais de amostragem em Londrina-PR no período de 02 a 24 de abril de 2020

\begin{tabular}{cccc}
\hline & Conc. Máxima & Conc. Mínima & Conc. Média \\
\hline BC UTFPR & 0,98 & 0,19 & 0,53 \\
$\mathrm{BC}_{2,5}-$ MHL & 1,51 & 0,33 & 0,76 \\
$\mathrm{BC}_{1,0}-$ MHL & 1,19 & 0,36 & 0,66 \\
\hline
\end{tabular}

Fonte: Dos autores.

\section{CONCLUSÃO}

Foram obtidas 42 amostras no MHL e 39 no TOU, onde estas apresentaram concentrações medias de $\mathrm{MP}_{2,5}$ e $\mathrm{MP}_{1,0}$ respectivamente iguais a 18,3 e $16,0 \mu \mathrm{g} \mathrm{m}^{-3}$ no MHL e 14,3 e 17,4 $\mu \mathrm{g} \mathrm{m}^{-3}$ no TOU. No MHL a fração de 1,0 micrometro do MP corresponde a $87 \%$ da fração de 2,5 micrometros, enquanto no TOU esta proporção é de $82 \%$. Os resultados obtidos no TOU apresentaram uma pequena diminuição na concentração de $\mathrm{MP}_{2,5}$ se comparado a trabalhos anteriores, entretanto, a razão entre $\mathrm{MP}_{2,5}$ e $\mathrm{MP}_{1,0}$ obteve um aumento de $1 \%$.

O BC obteve um valor menor no sítio amostral da UTFPR quando se comparado ao MHL, pois encontra-se em um local mais afastado na cidade. Na UTFPR a média observada para o BC foi de $0,53 \mu \mathrm{g} \mathrm{m}^{-3}$ enquanto no MHL foi de 0,76 e $0,66 \mu \mathrm{g} \mathrm{m}^{-3}$ para o $\mathrm{BC}_{2,5}$ e $\mathrm{BC}_{1,0}$, respectivamente. Observou-se em ambos os pontos de amostragem o aumento da concentração do $\mathrm{BC}$ subsequente ao relaxamento das medidas restritivas adotadas na cidade.

Os analitos analisados neste trabalho tiveram um acréscimo na concentração após o relaxamento das restrições. O MP teve um aumento médio de 31,5 e 27,4\% nas frações de 1,0 e 2,5 respectivamente no ambiente externo. No TOU a variação foi de 40,1 e 34,2\% para o $\mathrm{MP}_{1,0}$ e $\mathrm{MP}_{2,5}$ respectivamente.

As medidas adotadas pela administração da cidade de Londrina, para o combate ao avanço da COVID-19, contribuíram diretamente para a diminuição dos poluentes avaliados neste estudo. A interrupção das atividades industriais e do comercio implicaram na diminuição das fontes de emissão de poluentes atmosféricos. 


\section{REFERÊNCIAS}

APPLE MOBILITY TRENDS. Disponível em:

https://www.apple.com/covid19/mobility.

BARRETO, C. OMS declara doença pelo novo coronavírus como pandemia. Portal PEBMED, Rio de Janeiro, 11 de Mar. de 2020. Disponível em:

https://pebmed.com.br/oms-declara-doenca-pelo-novo-coronavirus-como-pandemia. Acesso em: 15/11/2020.

BEAL, A.; BUFATO, C. A.; ALMEIDA, D. S. de; SQUIZZATO, R.; ZEMIANI, A.; VERNILO, N.; BATISTA, C. E.; SALVADOR, G.; BORGES, D. L.G.; SOLCI, M. C. Inorganic Chemical Composition of Fine Particulates in Medium-Sized Urban Areas: a case study of brazilian cities. Aerosol And Air Quality Research, v. 17, n. 4, p. 920932, 2017. Taiwan Association for Aerosol Research.

http://dx.doi.org/10.4209/aaqr.2016.07.0317.

BEBER, L.C.C; GEWEHR, D.M.; CECCONELLO, L.; SULZBACHER, M. M.; HECK, T.G.; BERLEZI, E.M. Fatores de risco para doenças respiratórias em crianças brasileiras: Revisão Integrativa. p. 26-38, 2020. https://doi.org/10.33362/ries.v9i1.1660

DANTAS, G.; SICILIANO, B.; FRANÇA, B.; DA SILVA, C.M.; ARBILLA, G. The impact of COVID-19 partial lockdown on the air quality of the city of Rio de Janeiro, Brazil. Science of the Total Environment, vol. 729, p. 139085, 2020. DOI 10.1016/j.scitotenv.2020.139085. Disponível em: https://doi.org/10.1016/j.scitotenv.2020.139085.

DA SILVA, C.; LEMOS, B.; PINTO, J.; AMADOR, I.; SOLCI, M. Black Carbon Associated to PM1.0 and PM2.5: Mass Variation due to Combustion of Biodiesel/Diesel Blends (B5, B6, B7 and B8). Journal of the Brazilian Chemical Society, v. 30, p. 786-792, 2018. http://dx.doi.org/10.21577/0103-5053.20180209

DOMINGO, J.L.; ROVIRA, J. Effects of air pollutants on the transmission and severity of respiratory viral infections. Environmental Research, vol. 187, p. 109650, 2020. DOI 10.1016/j.envres.2020.109650. Disponível em: https://doi.org/10.1016/j.envres.2020.109650

FREITAS, A. M.; SOLCI, M. C. Caracterização do MP10 e MP2,5 e distribuição por tamanho de cloreto, nitrato e sulfato em atmosfera urbana e rural de Londrina. Química Nova, v. 32, n. 7, p. 1750-1754, 2019.

Google LLC. Google COVID-19 Community Mobility. Reports.https://www.google.com/covid19/mobility/ Acesso em: 10/04/2021

IAPAR IDR-PARANÁ - INSTITUTO AGRONÔMICO DO PARANÁ. Disponível em: http://www.idrparana.pr.gov.br/Pagina/Agrometeorologia-e-Clima

IBGE - INSTITUTO BRASILEIRO DE GEOGRAFIA E ESTATÍSTICA. Disponível em: https://www.ibge.gov.br/cidades-e-estados/pr/londrina.html/

INMET - INSTITUTO NACIONAL DE METEOROLOGIA. Disponível em: 
https://portal.inmet.gov.br/

INSTITUTO AMBIENTAL DO PARANÁ (IAP), INVENTÁRIO ESTADUAL DE EMISSÕES ATMOSFÉRICAS DE POLUENTES (MP, CO, NOX, SOX) E PROPOSTA PARA REVISÃO E AMPLIAÇÃO DA REDE DE MONITORAMENTO DA QUALIDADE DO AR DO ESTADO DO PARANÁ, 2013. Disponível em: http://www.iap.pr.gov.br/arquivos/File/Monitoramento/INVENTARIO/INVENTARIO_ ESTADUAL_DE_EMISSOES_ATM_versaofinal.pdf

KRECL, P.; TARGINO, A.C.; KETZEL, M.; CIPOLI, Y.; CHARRES, I. Potential to reduce the concentrations of short-lived climate pollutants in traffic environments: A case study in a medium-sized city in Brazil. Transportation Research Part D:

Transport and Environment, vol. 69, p. 51-65, 2019. DOI 10.1016/j.trd.2019.01.032. Disponível em: https://doi.org/10.1016/j.trd.2019.01.032.

LE QUÉRÉ, C.; JACKSON, R.B.; JONES, M.W.; SMITH, A.J.P.; ABERNETHY, S.; ANDREW, R.M.; DE-GOL, A.J.; WILLIS, D.R.; SHAN, Y.; CANADELL, J.G.; FRIEDLINGSTEIN, P.; CREUTZIG, F.; PETERS, G.P. Temporary reduction in daily global CO2 emissions during the COVID-19 forced confinement. Nature Climate Change, vol. 10, no. 7, p. 647-653, 2020. https://doi.org/10.1038/s41558-020-0797-x.

LELIEVELD, J.; POZZER, A.; PÖSCHL, U.; FNAIS, M.; HAINES, A.; MÜNZEL, T. Loss of life expectancy from air pollution compared to other risk factors: A worldwide perspective. Cardiovascular Research, vol. 116, no. 11, p. 1910-1917, 2020. https://doi.org/10.1093/cvr/cvaa025.

MANIGRASSO, M.; PROTANO, C.; GUERRIERO, E.; VITALI, M.; AVINO, P. May SARS-CoV-2 Diffusion be favoured by Alkaline Aerosols and Ammonia Emissions?

Atmosphere, vol. 11, p. 1-10, 2020. https://doi.org/10.3390/atmos11090995.

MARTELlETTI, L.; MARTELlETTI, P. Air Pollution and the Novel Covid-19 Disease: a Putative Disease Risk Factor. SN Comprehensive Clinical Medicine, vol. 2, p. 383-387, 2020. https://doi.org/10.1007/s42399-020-00274-4.

MINISTÉRIO DA SAÚDE. Disponível em: https://covid.saude.gov.br/

NAKADA, L.Y.; URBAN, R.C. COVID-19 pandemic: Impacts on the air quality during the partial lockdown in São Paulo state, Brazil. Science of the Total Environment, vol. 730, p. 139087, 2020. DOI 10.1016/j.scitotenv.2020.139087. Disponível em: https://doi.org/10.1016/j.scitotenv.2020.139087.

NARDOCCI, A.C.; FREITAS, C.U.; PONCE DE LEON, A.C.M.; JUNGER, W.L.; GOUVEIA, N. Poluição do ar e doenças respiratórias e cardiovasculares: estudo de séries temporais em Cubatão, São Paulo, Brasil. Cadernos de Saúde Pública, vol. 29, p. 1867-1876, 2013. https://doi.org/10.1590/0102-311X00150012

RAJAGOPALAN, S.; BROOK, R.D. Air pollution and type 2 diabetes: Mechanistic insights. Diabetes, vol. 61, p. 3037-3045, 2012. https://doi.org/10.2337/db12-0190.

SANTOS, D.R.F.; MANTOVANI, I.S.; SOUZA, J.; SOLCI, M.C. SAZONALIDADE DO MATERIAL PARTICULADO FINO E BLACK CARBON NO AR AMBIENTE 
DE LONDRINA/PARANÁ. Brazilian Journal of Development, vol 6, p. 8/406984086, 2020. https://doi.org/10.34117/bjdv6n10-736

SINIMBÚ, F. Saúde confirma primeiro caso provável de coronavírus em Curitiba e espera exames de um segundo. Bem Paraná, Paraná, 11 de Mar. de 2020. Disponível em: < https://www.bemparana.com.br/noticia/curitiba-registra-primeiro-caso-provavelde-coronavirus\#.X7Gz2mhKiUk>. Acesso em: 15/11/2020

UNIVERSIDADE ESTADUAL DE LONDRINA. Ato executivo n 22/2020, de 16 de março de 2020. Dispõe sobre a suspensão das atividades da UEL. 2020. Disponível em: http://www.uel.br/com/agenciaueldenoticias/index.php?arq=ARQ_not\&id=30122. Acesso em: 07/06/2020.

\section{Recebido em: 10/10/2021}

Aprovado em: 10/11/2021

Publicado em: 12/11/2021 